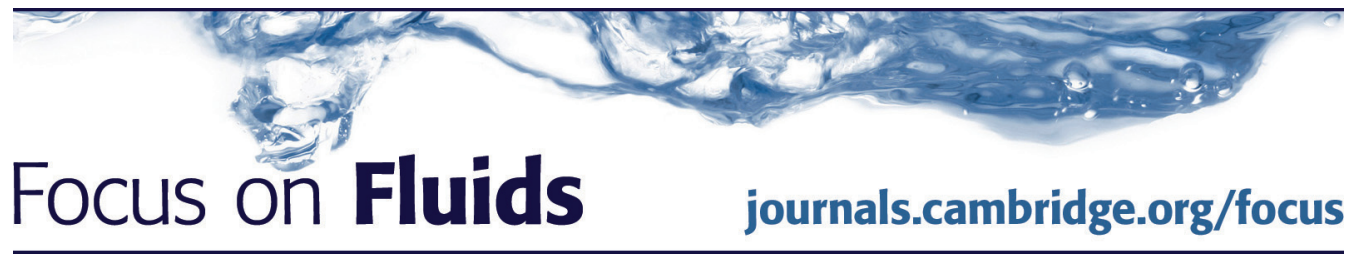

\title{
When is high Reynolds number shear flow not turbulent?
}

\author{
Steven A. Balbus $\dagger$ \\ Astrophysics, Department of Physics, \\ University of Oxford, Denys Wilkinson Building, \\ Keble Road, Oxford OX1 3RH, UK
}

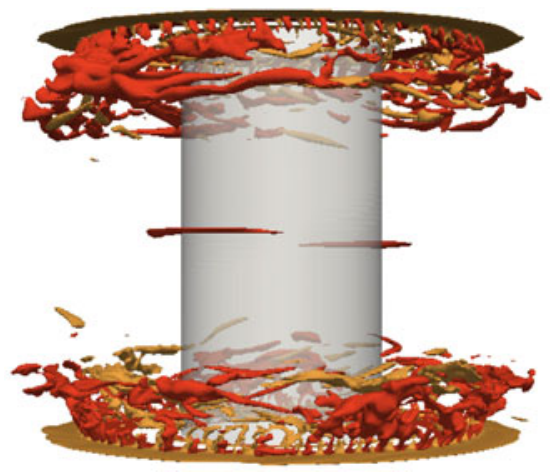

Rotating flow in which the angular velocity decreases outward while the angular momentum increases is known as 'quasi-Keplerian'. Despite the general tendency of shear flow to break down into turbulence, this type of flow seems to maintain stability at very large Reynolds number, even when nonlinearly perturbed, a behaviour that strongly influences our understanding of astrophysical accretion discs. Investigating these flows in the laboratory is difficult because secondary Ekman flows, caused by the retaining Couette cylinders, can become turbulent on their own. A recent high Reynolds number numerical study by Lopez \& Avila (J. Fluid Mech., vol. 817, 2017, pp. 21-34) reconciles apparently discrepant laboratory experiments by confirming that this secondary flow recedes toward the axial boundaries of the container as the Reynolds number is increased, a result that enhances our understanding of nonlinear quasi-Keplerian flow stability.

Key words: boundary layer stability, rotating turbulence, transition to turbulence

\section{Introduction}

Accretion discs are a key stage in the formation of galaxies, stars and planets, reservoirs that allow gas, via the presence of turbulence, to shed angular momentum before alighting onto the central object. What is origin of the turbulence? The standard linear Rayleigh criterion for rotational stability (specific angular momentum increasing radially outward) is well satisfied, prompting early studies to argue that the combination of shear and large Reynolds numbers would render any rotating flow, in particular the Keplerian rotation of interest, nonlinearly unstable (e.g. Shakura \& Sunyaev 1973).

Anti-cyclonic rotating flows for which the specific angular momentum increases radially outward while the angular velocity decreases are known as quasi-Keplerian $(\mathrm{qK})$. The nub of the problem is that when the stabilisation of the Coriolis force

$†$ Email address for correspondence: steven.balbus@physics.ox.ac.uk 
outweighs the Kelvin-Helmholtz-like shear destabilisation, qK flow does not rapidly break down like a non-rotating shear layer; it is much more stable. As a practical matter, the 1991 discovery (Balbus \& Hawley 1991) that weak and generally unavoidable magnetic fields are sufficient to completely destabilise Keplerian rotation addressed the immediate problem of the origin of internal turbulence in discs with even a small ionisation fraction. But even this modest requirement is not always met at all times and locations in protoplanetary discs, an important subclass. There has therefore been a longstanding effort to understand whether nonlinear, 'subcritical' instabilities might somehow be involved in the breakdown to turbulence, the stabilising Coriolis force notwithstanding.

Investigations of the nonlinear stability of $\mathrm{qK}$ flow have been based on perceived analogies to subcritical transitions that have been observed in laboratory experiments (e.g. Coles 1965). However, qK flow is in a very different regime, and numerical simulations consistently yield stable results for these flows. Subtle effects may be at play here, possibly triggered at very small scales, so the finite grid (or series termination in a spectral code) is a concern. Laboratory experiments are thus of interest as direct probes of flow stability and as a point of calibration for numerical codes.

The most obvious difference between an astrophysical accretion disc and a laboratory experiment is the container that holds the fluid. Sides and endcaps, absent in a real disc, produce boundary layers. The ensuing secondary flows are known as Ekman circulation. In an experiment designed to focus on the onset of turbulence, one must be certain that any breakdown of laminar flow is associated not with the Ekman circulation, but with the primary shear in the bulk of the fluid. This sets the stage for the numerical study described by Lopez \& Avila (2017).

\section{Overview}

In the original Couette experiment of $\mathrm{Ji}$ et al. (2006), the endcaps were split into two independently rotating rings, in order to minimise the viscous stress and ensuing Ekman circulation. Ji et al. found no evidence that $\mathrm{qK}$ flow broke down into turbulence at a shear Reynolds number (hereafter $R_{s}$ ) up to $10^{6}$, by far the best measured constraint. This was based on the absence of any increase in the Reynolds stress, a signature of turbulent flow. Another experimental approach, without split endcaps but of greater vertical extent than $\mathrm{Ji}$ et al., tried to isolate and study the midsection of the apparatus. Paoletti \& Lathrop (2011) found an increase in the outer torque (presumably via a heightened Reynolds stress) for similar $R_{s}$ values. Both results could not be correct. Or could they?

Avila (2012) undertook direct numerical simulations of these set-ups, and found that both were prone to secondary turbulent Ekman circulations for $R_{s} \sim 10^{3}$. This accounted for the Paoletti \& Lathrop (2011) findings, but seemed inconsistent with the null results of Ji et al. (2006).

Edlund \& Ji (2014) revisited the original Ji et al. (2006) investigation in a modified experimental set-up, and found that $\mathrm{qK}$ flow remained stable, even when strongly perturbed. They argued that the three orders-of-magnitude difference between the $R_{S}$ of Avila's numerical simulations $\left(\sim 10^{3}\right)$ and that of the laboratory set-up $\left(\sim 10^{6}\right)$ was important: more than surpassing a threshold value is involved. Edlund \& Ji (2015) followed with a laboratory study and model of how departures from ideal Couette flow change with $R_{s}$.

The recent study of Lopez \& Avila (2017) has succeeded in carrying out numerical simulations of the laboratory configurations of Edlund \& Ji (2014) for $R_{s}$ approaching 


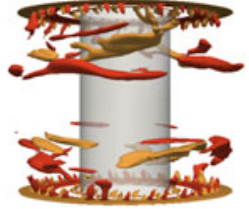

$R_{s}=9655$

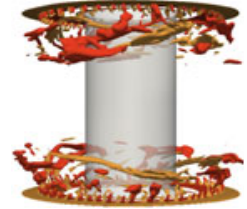

$R_{s}=19310$

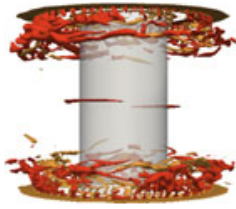

$R_{s}=32180$

FIgURE 1. Recession of radial velocity isosurfaces with increasing $R_{s}$ toward axial boundaries. See text for further details.

$10^{5}$, an order of magnitude below those of the laboratory, but beyond previous numerical studies of laboratory experiments. The role of viscosity is double edged. A large $R_{s}$ lowers small-scale dissipation, making it easier to sustain turbulent fluctuations. But heightened viscous stresses at smaller $R_{s}$ are a source of correlations in the radial and azimuthal velocity fluctuations, which allow the flow to extract free energy from the shear, making it easier to nourish turbulence. Lopez \& Avila find that at lower $R_{s}$ values, secondary Ekman circulation forms extensively, sometimes over the entire flow region. The secondary flows are prone to their own instabilities, unrelated to any hypothetical flow instability of the underlying $\mathrm{qK}$ profile. But at larger $R_{s}$, the turbulent flow recedes from the bulk of the zone of interest, confining itself to thin layers near the endcaps of the apparatus. The presumption then is that at yet larger $R_{s}$, the turbulence would be confined to very narrow boundary layers, leaving the bulk of the interior laminar. In brief, experiments and numerical simulations now seem to be broadly consistent with an absence of any nonlinear shear flow instability in $\mathrm{qK}$ flow.

Edlund \& Ji (2014) examined three configurations of a modified Couette apparatus. In the 'HTX' (hydrodynamic turbulence experiment) configuration, our focus here, the end cap is split into three annuli. Two of these rings are adjacent to the inner and outer cylinders, and spin at the same rate. The middle ring is independent. An 'optimal boundary condition' is defined by the requirement that the torques on the inner and outer cylinder be equal and opposite, while the endplate torques vanish. This corresponds to the ideal limit of axially infinite cylinders, and is obtained for a particular ratio of inner to outer cylinder rotation rate. The simulations of Lopez \& Avila (2017) were carried out under this condition.

The take-home message is summarised in figure 1. In the HTX configuration, as $R_{s}$ increases, the level of turbulent fluctuations decreases and recedes toward the endcaps. This is clear evidence that turbulence in Couette experiments is very sensitive to $R_{s}$ : low values, far from suppressing turbulence, are likely to trigger it throughout a broad region of the apparatus via secondary Ekman flows. As $R_{s}$ increases, the region of turbulence is confined to a diminishing boundary layer and the root mean square fluctuations decrease. In short, there is no compelling reason to believe that $\mathrm{qK}$ shear flow, in itself, is intrinsically unstable.

\section{The future}

The findings of Lopez \& Avila (2017) are broadly consistent with what is known about Ekman layers: their thickness scales inversely with a power of $R_{s}$, in classical applications an inverse square root. We see a clear demonstration of this trend in a numerical simulation of a realistic laboratory apparatus. This work heralds an 
expanded role for high quality numerical investigations of laboratory Couette flow, perhaps under a variety of different boundary conditions. At present, the laboratory is approximately an order of magnitude ahead in $R_{s}\left(10^{6}\right.$ versus $\left.10^{5}\right)$, and we may soon anticipate convergence.

Astrophysicists, note the focus here is reduced to the nature of Ekman circulation, not shear instability in qK flow. Whatever putative instabilities may be lurking in the bulk of the main flow (and there is scant evidence there are any), this flow is hardly in the same vein as simple shear, hypersensitive to small perturbations. Dynamicists who remain convinced that high Reynolds number shear flow of any sort is unstable must be prepared to dig below the level of the unavoidable secondary Ekman circulation. (Ever more powerful numerical simulations of free-boundary discs are an alternative route.)

Other dynamics, notably baroclinic rotation, may be a source of flow destabilisation, but the goal here is not instability per se. It is not even the onset of turbulence. It is a mode of enhanced internal angular momentum transport. This is of course what turbulence sometimes does - but not always. The key is an enhanced correlation in the radial and azimuthal velocity fluctuations. If the cause of the breakdown of laminar flow is the radial shear itself, this correlation is more likely to appear (as in the magneto-rotational instability), than if the cause is, say, an adverse entropy gradient or related vertical velocity gradient.

Fluid dynamicists, note there is a grand challenge problem here. All indicators seem to imply that large Reynolds number $\mathrm{qK}$ flow is truly nonlinearly stable. Can this be proven analytically? What valuable insights might emerge by even a partially successful attempt to do so?

\section{Acknowledgement}

I am grateful to the Royal Society and to STFC for financial support.

\section{References}

AvILA, M. 2012 Stability and angular-momentum transport of fluid flows between corotating cylinders. Phys. Rev. Lett. 108, 124501.

Balbus, S. A. \& Hawley, J. F. 1991 A powerful local shear instability in weakly magnetized disks. Astrophys. J. 376, 214-222.

Coles, D. 1965 Transition in circular Couette flow. J. Fluid Mech. 21, 385-421.

EdLund, E. M. \& Ji, H. 2014 Nonlinear stability of laboratory quasi-Keplerian flows. Phys. Rev. E 89, 021004.

EdLund, E. M. \& JI, H. 2015 Reynolds number scaling of the influence of boundary layers on the global behaviour of quasi-Keplerian flows. Phys. Rev. E 92, 043005.

Ji, H., Burin, M., Schartman, E. \& Goodman, J. 2006 Hydrodynamic turbulence cannot transport angular momentum effectively in accretion disks. Nature 444, 343-346.

Lopez, J. M. \& Avila, M. 2017 Boundary-layer turbulence in experiments on quasi-Keplerian flows. J. Fluid Mech. 817, 21-34.

Paoletti, M. S. \& Lathrop, D. P. 2011 Angular momentum transport in turbulent flow between independently rotating cylinders. Phys. Rev. Lett. 106, 024501.

ShakURA, N. I. \& SUNYAEV, R. A. 1973 Black holes in binary systems. Observational appearance. Astron. Astrophys. 24, 337-355. 